\title{
Cosmic rays from active galactic nuclei
}

\author{
E.G.Berezhko ${ }^{1}$ \\ berezhko@ikfia.ysn.ru
}

\begin{abstract}
Cosmic ray (CR) acceleration at the shock created by the expanding cocoons around active galactic nuclei (AGNs) is studied. It is shown that above the energy $10^{18} \mathrm{eV}$ the overall energy spectrum of CRs, produced during the AGN evolution and released in the intergalactic space, has the form $N \propto \epsilon^{-\gamma}$, with $\gamma \approx 2.6$, which extends up to $\epsilon_{\max } \sim 10^{20} \mathrm{eV}$. It is concluded that cocoons shocks have to be considered as a main source of extragalactic CRs, which together with Galactic supernova remnants provide the observed CR spectrum.
\end{abstract}

Subject headings: cosmic rays — acceleration of particles — shock waves supernova remnants - galaxies: active

\section{Introduction}

The overall origin of cosmic rays (CRs) is still an unresolved problem in astrophysics. The understanding of $\mathrm{CR}$ origin requires the determination of the astrophysical objects, that are the CR sources, and of the appropriate acceleration processes, that form the CR spectrum in these objects. In this regard, during the last several years considerable progress has been achieved in this field, both experimentally and theoretically. Recently the steepening of the CR spectrum above $3 \times 10^{19} \mathrm{eV}$ was established in the HiRes (Bergman et al. 2007) and Auger (Yamamoto et al. 2007) experiments. It presumably corresponds to the so-called GreizenZatsepin-Kuzmin (GZK) cutoff, caused by CR energy losses in their interactions with the microwave background radiation. This is evidence that the highest energy part of the CR spectrum is of extragalactic origin. It was also recently demonstrated (Berezhko \& Völk 2007) that the CRs with energies up to $\epsilon \sim 10^{17} \mathrm{eV}$ can be produced in supernova remnants (SNRs).

The main reason why SNRs are usually considered as the CR source is a simple argument about the energy required to sustain the Galactic CR population against loss by escape,

\footnotetext{
${ }^{1}$ Yu.G. Shafer Institute of Cosmophysical Research and Aeronomy, 31 Lenin Ave., 677980 Yakutsk, Russia
} 
nuclear interactions and ionization energy loss. Supernovae have enough power to drive the $\mathrm{CR}$ acceleration. The high velocity ejecta produced in the supernova explosion interacts with the ambient medium to produce a strong blast wave, which may accelerate a small suprathermal fraction of the ambient plasma to high energies. The only theory of particle acceleration which is sufficiently well developed and specific to allow quantitative model calculations is diffusive acceleration (Krymskv 1977; Bell 1978) applied to the strong outer shock associated with SNRs (e.g. see Berezhko \& Krymsky 1988; Berezhko 2005, 2008, for review). Considerable efforts have been made during the last years to empirically confirm the theoretical expectation that the main part of CRs indeed originates in SNRs. Theoretically, progress in the solution of this problem has been due to the development of a kinetic nonlinear theory of diffusive shock acceleration (Berezhko et al. 1996). This theory includes all the most relevant physical factors, essential for the SNR evolution and CR acceleration in a SNR and it is able to make quantitative predictions of the expected properties of CRs produced in young SNRs and their nonthermal radiation. Applied to individual young SNRs (see Berezhko 2005, 2008, for reviews) this theory has successfully explained many observed SNR properties. Making use of the observed synchrotron emission spectrum from radio to $\mathrm{X}$-ray frequencies it permits the determination of the degree of magnetic field amplification, a process advocated earlier from plasma simulations (Lucek \& Bell 2000; Bell \& Lucek 2001), which leads to the concentration of the highest-energy electrons in a very thin shell just behind the shock. Such observed filamentary structures have been observed in hard Xrays (Vink \& Laming 2003; Long et al. 2003; Bamba et al. 2003; Parizot et al. 2006) and are nowadays used as a second independent method to infer the magnitude of the amplified field (see Berezhko et al. 2003, for interpretation).

It has been demonstrated, that magnetic field amplification leads to considerable increase of the maximal energy of CRs accelerated in SNRs and that the observed CR energy spectrum can be well represented by two components (Berezhko \& Völk 2007). The first one, $J^{g}(\epsilon)$, dominant up to $10^{17} \mathrm{eV}$, consists of CRs produced in Galactic SNRs, whereas the second, $J^{e g}(\epsilon)$, is produced in extragalactic sources. This so called dip scenario (Aloisio et al. 2007) requires a relatively steep CR spectrum produced in extragalactic sources, $J_{\mathrm{s}}^{e g} \propto \epsilon^{-\gamma}$ with $\gamma=2.55-2.75$ at energies $\epsilon>10^{18} \mathrm{eV}$. It has been demonstrated that peculiarities in the CR spectrum - the so-called "dip" structure at $\epsilon \sim 10^{19} \mathrm{eV}$ and a GZK cutoff at $\epsilon>3 \times 10^{19} \mathrm{eV}$ (Aloisio et al. 2007) - produced due to CR interaction with the cosmic microwave background radiation on their way from the source to the observer are very consistent with the experiment.

Below we consider CR acceleration at the shock created by the expanding cocoon around active galactic nuclei (AGNs). Compared with previous considerations (Norman et al. 1995) magnetic field amplification is taken into account, which provides $\mathrm{CR}$ acceleration with an 
appropriate spectrum up to the energy $10^{20} \mathrm{eV}$. Therefore the cocoon shocks have to be considered as a main source of extragalactic CRs, which together with Galactic supernova remnants presumably provide the observed CR spectrum.

\section{Results and discussion}

Astrophysical objects, which are considered as potential extragalactic sources of ultra high energy CRs, should fulfill a number of requirements. First, they should have sufficiently high energy output, not less than $L_{\mathrm{p}} \sim 2 \times 10^{45}-3.5 \times 10^{46} \mathrm{erg}_{\mathrm{Mpc}}^{-3} \mathrm{yr}^{-1}$ depending on the form of CR spectrum that they produce (Berezinsky et al. 2006).

Given the energy requirements, AGNs (e.g. Berezinskv et al. 2006) and gamma-ray bursts (GRBs) (Milgrom \& Usov 1995; Waxman 1995; Vietri 1995) are considered as potential extragalactic sources of the ultra-high-energy CRs. However, as Berezinsky et al. (2006) argued, the energy output of GRBs has a serious problem if they are considered as the main source of the extragalactic CRs.

The second requirement to the extragalactic CR sources is their ability to produce a power-law CR spectrum up to the maximal energy, which for protons should be at least as large as $\epsilon_{\max }=10^{20} \mathrm{eV}$, which is well above the GZK cutoff. Particle acceleration at relativistic shocks in AGNs (Achterberg et al. 2000; Rachen \& Biermann 1993) and GRBs (Milgrom \& Usov 1995; Waxman 1995; Vietri 1995) are discussed as appropriate acceleration processes. However, simulations of CR shock acceleration (Niemiec et al. 2006) have demonstrated a low efficiency in the case of relativistic shocks.

The relativistic jet in AGNs is surrounded by the hot cocoon, which expands into the surrounding intergalactic medium with supersonic speed (e.g. Ferrari 1998). Since the essential fraction of the jet energy is transformed into the internal energy of the background medium due to the outermost nonrelativistic shock, driven by the cocoon overpressure, it is natural to suppose that a good part of this energy is represented by effectively accelerated CRs, like what takes place in SNRs. Since CR acceleration by nonrelativistic shocks has been very well studied one can obtain reliable estimates of the expected spectrum of CRs. First of all the maximal energy of CRs, accelerated at the expanded shock of size $R(t)$ and speed $V=d R / d t$, is determined by the expression (Berezhko 1996)

$$
\epsilon_{\max } \approx Z e B R V / c
$$

where $B$ is the upstream magnetic field, $c$ is the speed of light, $e$ is the proton charge and $Z$ is the charge number of CR nuclei. This expression consistently takes into account the influence of all the most essential physical factors restricted the maximal CR energy, which are the 
adiabatic cooling of CRs in the downstream region and the shock deceleration followed by the CR escape from the shock vicinity into outer space (Berezhko 1996).

Magnetic field near the shock front, as was established for all young SNRs (Berezhko 2005, 2008), is strongly amplified nonlinearly by CRs up to the level

$$
B^{2} /(8 \pi) \approx 3 \times 10^{-3} \rho V^{2}
$$

which is presumably also appropriate for extragalactic shocks. Here $\rho$ is the external gas density. Note that the relation $B \propto V$ following from Eq.(2) is indeed theoretically expected for the shock speeds $V<10^{4} \mathrm{~km} \mathrm{~s}-1$ (Pelletier et al. 2006). Due to the nonresonantly amplified magnetic field, as was originally shown by Bell (2004) (see also Pelletier et al. 2006), at higher shock speed $V>10^{4} \mathrm{~km} \mathrm{~s}-1$ the expected relation is $B \propto V^{3 / 2}$, which leads to larger magnetic field strength compared with the value given by Eq.(2). Due to this fact CR maximal energy $\epsilon_{\max }$ can be a few times larger compared with our estimate below. However this does not play a critical role because even our estimate gives $\epsilon_{\max } \approx 10^{20} \mathrm{eV}$, which is well above GZK cutoff.

The outer cocoon shock expands with the speed (e.g. Ferrari 1998)

$$
V \approx\left[L_{\mathrm{j}} /\left(\rho V_{\mathrm{h}}\right)\right]^{1 / 4} t^{-1 / 2}
$$

where $L_{\mathrm{j}}$ is the mechanical luminosity of the jet and $V_{\mathrm{h}}$ is the hot spot or the jet head speed. Substituting the magnetic field value following from Eq.(2), the shock speed (3) and the shock size $R=2 V t$ into the equation (1), we have

$$
\begin{aligned}
\epsilon_{\max } \approx & 10^{20} Z\left(\frac{L_{\mathrm{j}}}{10^{46} \mathrm{erg} / \mathrm{s}}\right)^{3 / 4}\left(\frac{N_{\mathrm{g}}}{10^{-4} \mathrm{~cm}^{-3}}\right)^{-1 / 4} \\
& \times\left(\frac{V_{\mathrm{h}}}{10^{10} \mathrm{~cm} / \mathrm{c}}\right)^{-1 / 4}\left(\frac{l_{\mathrm{j}}}{1 \mathrm{kpc}}\right)^{-1 / 2} \mathrm{eV}
\end{aligned}
$$

where $l_{\mathrm{j}}=V_{\mathrm{h}} t$ is the jet length. According to this expression, during the evolutionary epochs corresponding to the jet length from $l_{\mathrm{j}}=1 \mathrm{kpc}$ to $l_{\mathrm{j}}=10 \mathrm{Mpc}$ the cocoon shock produces a power-law proton spectrum which extends up to the maximal energy from $\epsilon_{\max }=10^{18} \mathrm{eV}$ to $\epsilon_{\max }=10^{20} \mathrm{eV}$. Thus we conclude, that AGNs fulfill also the second requirement for extragalactic CR sources: protons, the main kind of ions in the space plasma, are effectively accelerated at least up to the energy $\epsilon_{\max }=10^{20} \mathrm{eV}$. Heavier ions are expected to be accelerated to $Z$ times higher maximal energy. However, since the GZK cutoff energy of nuclei $\epsilon_{\mathrm{GZK}}^{A}<\epsilon_{\mathrm{GZK}}$ for the atomic number $A>1$ is lower then that for protons $\epsilon_{\mathrm{GZK}} \approx$ $5 \times 10^{19} \mathrm{eV}$ (Berezinsky et al. 2006), the predicted dependence $\epsilon_{\max } \propto Z$ is difficult to establish experimentally. 
The most realistic possibility of finding the signature of the acceleration process of ultrahigh-energy CRs is the experimental study of CR composition at energies $\epsilon>10^{17} \mathrm{eV}$. If extragalactic CRs are indeed produced by large-scale nonrelativistic shocks their composition will have the following peculiarity. Inside and near the source, CR composition corresponds to the composition of the intergalactic gas with substantial enrichment by heavy elements, like with Galactic CR versus interstellar medium compositions (e.g. Berezhko et al. 1996). If compositions of intergalactic and interstellar plasmas are not very different, then the expected source $\mathrm{CR}$ composition is characterised by the mean atomic number $\langle A\rangle \approx 5$, like Galactic CR components. Compared with this source composition, the observed composition is significantly distorted at almost all energies due to propagation effects in the intergalactic medium and in the Galactic wind. Only at the energy $\epsilon_{*} \approx 2 \times 10^{18} \mathrm{eV}$ are these compositions roughly the same because it is just below the lowest GZK cutoff energy $\epsilon_{\mathrm{GZK}}^{\mathrm{He}} \approx 4 \times 10^{18} \mathrm{eV}$ and the modification of the proton spectrum at this energy is still not very large (Berezinsky et al. 2006). Since CR diffusion mobility for given energy $\epsilon$ is lower for larger $Z$, heavier CRs undergo stronger modification (depression) during their propagation from the sources to the observer. Due to this effect composition of extragalactic CRs is expected to become progressively lighter with the decrease of their energy at $\epsilon\left\langle\epsilon_{*}\right.$. At higher energies, $\epsilon>\epsilon_{*}$, due to the dependence of the GZK cutoff energy $\epsilon_{\mathrm{GZK}} \propto A$ on the nuclear mass number $A$, CRs become progressively heavier so that the peak value of $\langle A\rangle$ is expected at the beginning of the GZK cutoff for iron, which is at $\epsilon \approx 10^{19} \mathrm{eV}$ (Berezinsky et al. 2006). At such a high energies only protons and iron nuclei survive and the iron-to-proton ratio has a maximum value at the energy $\epsilon \approx 10^{19} \mathrm{eV}$, which provides a peak of $\mathrm{CR}$ mean mass very similar to the peak at $\epsilon \approx 10^{17} \mathrm{eV}$. To make a quantitative prediction of the expected ultra-high-energy CR composition, one has to perform a detailed consideration, which will be done in a subsequent paper.

Note that the expression (4) is based on the amplified magnetic field value (2) and therefore does not depend on the assumed value of intergalactic magnetic field in contrast to the earlier estimate of $\epsilon_{\max }$ (Norman et al. 1995). Note also that as is seen from the Eq.(4), maximal CR energy only slightly depends on external gas density and the jet head speed. The values of mechanical luminosity $L_{\mathrm{j}}$ and the jet length $l_{\mathrm{j}}$, which determines the maximal CR energy, can be directly measured in the experiment.

The third requirement to $\mathrm{CR}$ sources is related to the form of the $\mathrm{CR}$ spectrum. The form of the resulting $\mathrm{CR}$ spectrum produced during the whole evolution of the expanding shock is determined by three physical factors: (1) nonlinear shock modification due to the CR back-reaction, (2) adiabatic energy losses in the downstream region, and (3) diffusive CR escape from the shock vicinity into outer space. The existence of the CR escape phenomenon makes it possible to estimate the shape of the spectrum of the most energetic CRs produced 
during the source evolution. As was demonstrated analytically Berezhko \& Krymsky 1988; Berezhko 1996) and confirmed numerically (Berezhko et al. 1996), since the maximal energy $\epsilon_{\max }(t)$ of CRs accelerated by the expanding shock during its most active phase decreases with time $t$ due to shock deceleration, the most energetic particles with energy $\epsilon \sim \epsilon_{\max }(t)$ undergo diffusive escape from the shock vicinity into the surrounding space. If such behavior happens during the time period from $t_{1}$ to $t_{2}$, then at any given epoch $t_{1}<t<t_{2}$ all accelerated particles with energies $\epsilon>\epsilon_{\max }(t)$ have already escaped and inside the source there are only particles with $\epsilon \leq \epsilon_{\max }(t)$.

Note that the particle escape phenomenon, has two important aspects. First, the energy of escaped CRs determined for any evolutionary epoch by Eq.(1) does not depend any more on the adiabatic cooling inside the parent expanding source, like what happens with CRs of lower energies $\epsilon<\epsilon_{\max }(t)$. Second, the spectrum of the escaped CRs can be essentially different compared with the canonical spectrum $N \propto \epsilon^{-2}$, produced by strong nonrelativistic shock. To estimate the spectrum of escaped CRs one can use the simple relation (Berezhko \& Krymsky 1988)

$$
N(\epsilon) \epsilon d \epsilon \propto \rho V^{2} R^{2-\beta} d R
$$

which determines at any given phase $t$ the overall number of CRs $N(\epsilon)$ with highest energy $\epsilon \sim \epsilon_{\max }(t)$. Due to the hard self-consistent spectrum of CRs, produced by the strong shock, the main contribution to the CR energy content is given by the particles with highest energy $\epsilon \sim \epsilon_{\max }(t)$ (Berezhko et al. 1996). Therefore their energy content scales as the shock ram pressure $\rho V^{2}$ times the shock volume, as it is given by Eq.(5). Factor $R^{-\beta}$ describes the progressive slow decrease of $\mathrm{CR}$ acceleration efficiency due to the shock weakening. For the shock expansion law $R \propto t^{-\nu}$ relation (5) gives

$$
N(\epsilon) \propto \epsilon^{-\gamma} \text { with } \quad \gamma=1+(2-\beta) /(2 / \nu-3)
$$

In our case $\nu=1 / 2$ this gives $\gamma=3-\beta$. For the constant acceleration efficiency we have $\beta=0$ which gives $\gamma=3$. However, the shock deceleration is accompanied by the decrease of the acceleration efficiency. This effect is described by the amount of shock modification, which is characterized by the shock compression ratio, which for the case of a strong shock depends on the shock speed as $\sigma \propto V^{3 / 8}$ (Berezhko et al. 1996). Since in our case $V \propto 1 / R$ we have $\beta=3 / 8$ that gives $\gamma \approx 2.6$. Such a spectrum of extragalactic CRs at energies $\epsilon>10^{18} \mathrm{eV}$ corresponds very well to the experiment (Berezinsky et al. 2006).

Particles with energies $\epsilon \leq \epsilon_{\max }(t)$ at any given epoch are contained inside the source and have a spectrum close to $N \propto \epsilon^{-2}$. At a very late epoch when the outer shock becomes weak these particles will leave the source. Note however that this is already not important for 
the Galaxy, because the contribution of extragalactic CRs is expected to be low at energies $\epsilon<10^{18} \mathrm{eV}$ (Berezinsky et al. 2006).

Note that based on the data collected at the Auger experiment, a correlation between the arrival directions of CRs with energy above $6 \times 10^{19} \mathrm{eV}$ and the position of nearby AGNs has been found (Abraham et al. 2007), which strongly supports AGNs as prime candidates for the source of ultra-high-energy CRs.

\section{Summary}

Magnetic field amplification due to shock-accelerated CRs provides acceleration of CRs up to the energy $\epsilon_{\max } \sim 10^{20} \mathrm{eV}$ at the outer nonrelativistic shock created by the expanding cocoon around AGNs .

The expected CR spectrum formed due their escape from the expanding shock has a form $N \propto \epsilon^{-\gamma}$ with $\gamma \approx 2.6$ at energies $\epsilon>10^{18} \mathrm{eV}$, which fulfills quite well the requirements for extragalactic CR sources. Therefore AGNs have to be considered as a prime candidate for the sources of extragalactic CRs. In such a case the observed CRs consist of two components: Galactic CRs, produced in SNRs, and extragalactic CRs from AGNs.

The expected composition of ultra-high-energy CRs is characterised by two peaks in the energy dependence of the mean $\mathrm{CR}$ atomic number $\langle A(\epsilon)\rangle$. The first one at the energy $\epsilon \approx 10^{17} \mathrm{eV}$ corresponds to the very end of the Galactic CR component (Berezhko \& Völk 2007), whereas the second, at the energy $\epsilon \approx 10^{19} \mathrm{eV}$, is expected at the beginning of the GZK cutoff. The strong energy dependence of CR composition within the energy interval from $10^{17}$ to $10^{18} \mathrm{eV}$ is expected as a signature of the transition from Galactic to extragalactic CRs, whereas the detection of heavy CR composition at $\epsilon \approx 10^{19} \mathrm{eV}$ has to be considered as the signature of $\mathrm{CR}$ production by nonrelativistic shocks.

The work was supported by the Presidium of the RAS (program 16) and by the Russian Foundation for Basic Research (grants 06-02-96008 and 07-02-00221).

\section{REFERENCES}

Abraham, J. (Pier Auger Collaboration) 2007, Science, 318, 938

Achterberg, A., Gallant, Y.A., Kirk, J.G., \& Guthmann, A.W. 2000, MNRAS, 328, 393 
Aloisio, R., et al. 2007, Astropart. Phys., 27, 76

Bamba, A., Yamazaki, R., Ueno, M., \& Koyama, K. 2003, ApJ, 589, 827

Bell, A.R. 1978, MNRAS, 182, 147

Bell, A. R., \& Lucek, S.G. 2001, MNRAS, 321, 433

Bell, A.R. 2004, MNRAS, 353, 550

Berezhko, E.G. 1996, Astropart. Phys., 5, 367

Berezhko, E.G. 2005, Adv. Space Res., 35, 1031

Berezhko, E.G. 2008, Adv. Space Res., 41, 429

Berezhko, E.G., \& Krymsky, G.F. 1988, Soviet Phys.-Uspekhi, 12, 155

Berezhko, E.G., Elshin, V.K., \& Ksenofontov, L.T. 1996, JETPh, 82, 1

Berezhko, E. G., Ksenofontov, L. T., \& Völk, H. J. 2003, A\&A, 412, L11

Berezhko, E.G. \& Völk, H.J. 2007, Astrophys. J., 661, L175

Berezinsky, V.S., Gazizov, A., \& Grigorieva, S. 2006, Phys. Rev. D, 74, 043005

Bergman, D.R. et al. 2007 Nuclear Phys. B (Proc. Supl.), 165, 19

Ferrari, A. 1998, Annu. Rev. Astron. Astrophys., 36, 539

Krymsky, G.F. 1977, Soviet Phys. Dokl., 23, 327

Long, K.S., Reynolds, S.P., Raymond, J.C., et al. 2003, ApJ, 586, 1162

Lucek, S.G., \& Bell, A.R. 2000, MNRAS, 314, 65

Milgrom, M. \& Usov, V. 1995, ApJ, 449, L37

J. Niemiec, J., Ostrowski, M., \& Pohl, M. 2006, ApJ, 650, 1020

Norman, C.A., Melrose, D.B. \& Achterberg, A. 1995, ApJ, 454, 60

Parizot, E., Marcowith, A., Ballet, J., \& Gallant, Y.A. 2006, A\&A, 453, 387

Pelletier, G. Lemoine, M., \& Marcovith, A. 2006, A\&A, 453, 181

Rachen, J.P., \& Biermann, P. 1993, A\&A, 272, 161 
Vietri, M. 1995, ApJ., 453, 883

Vink, J., \& Laming, J.M. 2003, ApJ, 548, 758

Waxman, E. 1995, Phys. Rev. Let., 75, 386

Yamamoto, T. et al. arXiv:0707.2638v3[astro-ph](2007)

This preprint was prepared with the AAS IATEX macros v5.0. 